\title{
HOMOCLINIC ORBITS IN THE EULER PROBLEM OF TWO FIXED CENTERS
}

\author{
SEONGCHAN KIM
}

\begin{abstract}
We give a complete description of the shapes and the behavior of all homoclinic orbits in the Euler problem of two fixed centers.
\end{abstract}

\section{Contents}

1. Introduction

2. The molecule theory

3. The Euler problem of two fixed centers

4. Molecules of the Euler problem 9

5. The existence of homoclinic orbits

6. Proof of the theorem 10

References

\section{INTRODUCTION}

A homoclinic orbit, whose concept was introduced by Poincaré, is an orbit which is asymptotic to an unstable periodic orbit in both forward and backward time. In view of the Poincare section map, an unstable periodic orbit corresponds to a hyperbolic fixed point of this map and a homoclinic orbit is represented by a point whose orbit is doubly asymptotic to the hyperbolic fixed point.

In this paper, we study homoclinic orbits in the Euler problem of two fixed centers. This problem describes the motion of a point-mass under the influence of a Newtonian potential with two fixed attracting points. We refer to the two attracting bodies as the Earth and Moon and the pointmass as the satellite. This problem can be obtained from the planar circular restricted three-body problem by ignoring the centrifugal and Coriolis terms. That this system is integrable was discovered by Euler in 1760 .

We denote by $\mu \in(0,1)$ the mass ratio of the two massive bodies and locate the Earth and Moon at $E=(-1 / 2,0)$ and $M=(1 / 2,0)$, respectively. The describing Hamiltonian $H: T^{*}\left(\mathbb{R}^{2} \backslash\{E, M\}\right) \cong$ $\left(\mathbb{R}^{2} \backslash\{E, M\}\right) \times \mathbb{R}^{2} \rightarrow \mathbb{R}$ is given by

$$
H(q, p)=\frac{1}{2}|p|^{2}-\frac{1-\mu}{|q-E|}-\frac{\mu}{|q-M|} .
$$

Without loss of generality we may assume that $\mu \leq 1 / 2$, i.e., the Earth is stronger. The Hamiltonian has a unique critical point $L=(l, 0,0,0), l \in(-1 / 2,1 / 2)$, of Morse index 1 . It corresponds to the saddle point of the potential.

Given an energy level $H=c$, we define the Hill's region to be

$$
\mathcal{K}_{c}:=\pi\left(H^{-1}(c)\right) \subset \mathbb{R}^{2} \backslash\{E, M\},
$$

where $\pi:\left(\mathbb{R}^{2} \backslash\{E, M\}\right) \times \mathbb{R}^{2} \rightarrow \mathbb{R}^{2} \backslash\{E, M\}$ is the footpoint projection. In what follows, we only consider negative energies so that Hill's regions are bounded, namely the satellite is confined to a

Date: November 5, 2018. 

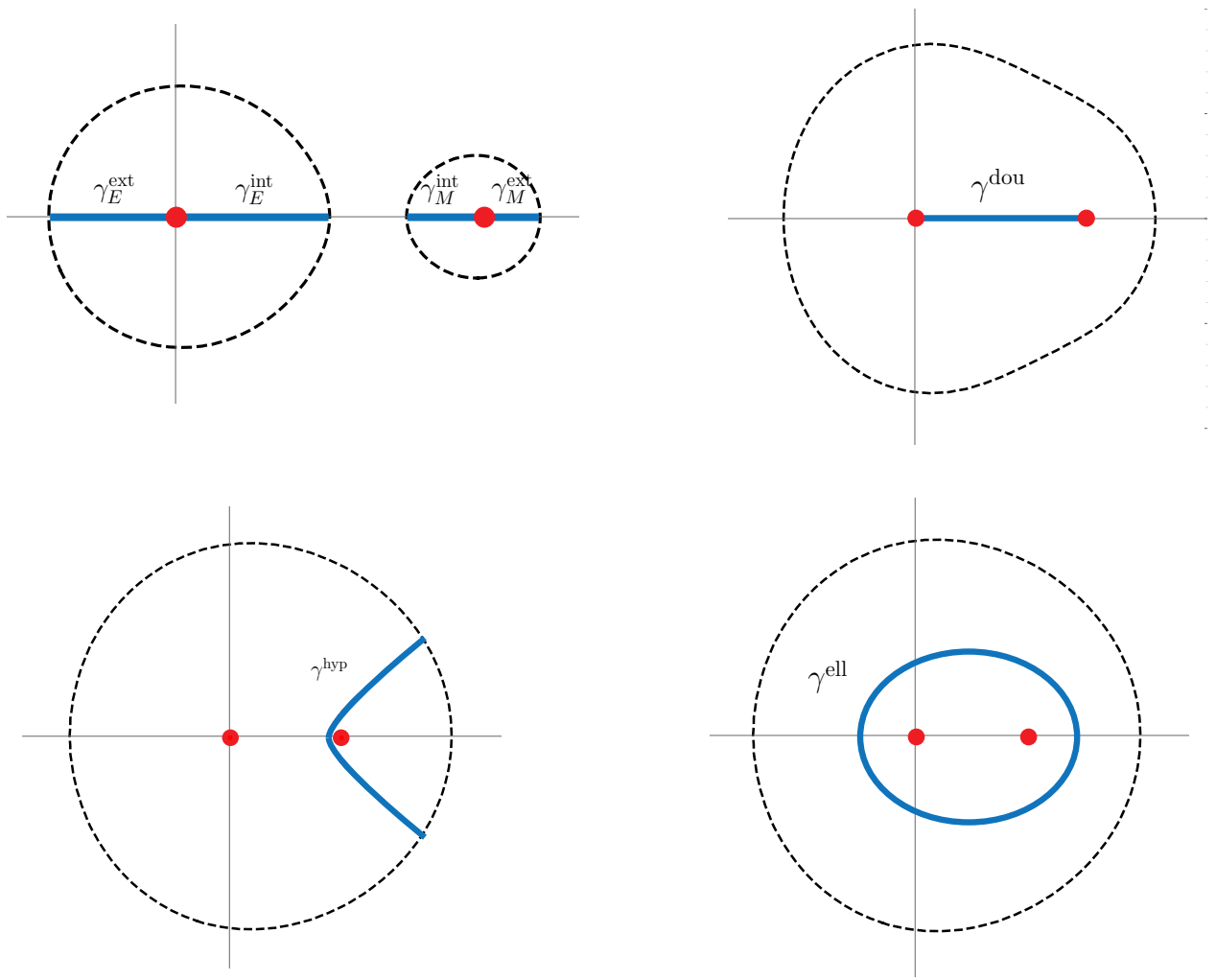

Figure 1. Critical orbits in the Euler problem

bounded region in the configuration space $\mathbb{R}^{2} \backslash\{E, M\}$. We denote by $c_{J}$ the critical energy level $H(L)=-1-2 \sqrt{\mu(1-\mu)}$. For $c<c_{J}$, the Hill's region consists of two connected components, where one of them is a neighborhood of the Earth and the other is a neighborhood of the Moon. We denote them by $\mathcal{K}_{c}^{E}$ and $\mathcal{K}_{c}^{M}$, respectively. For $c>c_{J}$, these two components become connected. The satellite then can move from the Earth to the Moon and vice versa. There are two further distinguished energy levels $c_{e}$ and $c_{h}$ at which the Liouville foliation changes, see Figure 7. Note that $c_{J}<c_{e}<c_{h}<0$ if $\mu \neq 1 / 2$ and $c_{J}<c_{e}<c_{h}=0$ if $\mu=1 / 2$.

For negative energies, there exist four types if $\mu \neq 1 / 2$ or three types if $\mu=1 / 2$ of regular regions. They are distinguished by five types if $\mu \neq 1 / 2$ or four types if $\mu=1 / 2$ of critical curves, see Figure 7. Each point on the critical curves represents one of five types of critical orbits: the interior and exterior collision orbits near each primary, the double-collision orbit, the hyperbolic orbit and the elliptic orbit, see Figure 1. The orbits of interest in this paper are the double-collision orbit, the hyperbolic orbit and the exterior collision orbit near the Moon: The double-collision orbit exists for $c>c_{J}$ and up to $c=c_{e}$ it is stable. However, passing the distinguished energy level $c=c_{e}$ it becomes unstable. The hyperbolic orbit, which exists for $c \in\left(c_{J}, c_{h}\right)$, is always unstable. Finally, the exterior collision orbit near the Moon exists for all negative energies and it is unstable if $\mu \neq 1 / 2$ and $c \in\left(c_{h}, 0\right)$. In other words, there exist three types of unstable periodic orbits. A natural question then arises: Do these unstable periodic orbits admit homoclinics? Since the Euler problem is integrable, the existence of homoclinics is well-known. In Section 5 , we recheck this well-known fact using the topological invariants of integrable Hamiltonian systems, which will be recalled in Section 2 ,

The main theorem of this paper concerns the behavior of homoclinics to each unstable periodic orbits: 
Theorem. The following do not depend on the choice of the mass ratio:

(i) For each $c \in\left(c_{J}, c_{h}\right)$, the hyperbolic orbit $\gamma^{\text {hyp }}$ admits homoclinics which collide with either the Earth or Moon. Moreover, all non-collision homoclinic orbits to $\gamma^{\text {hyp }}$ rotate around one of the primaries precisely once, see Figure 2

(ii) Fix $c \in\left(c_{e}, 0\right)$ and consider the double-collision orbit $\gamma^{\text {dou }}$. All homoclinic orbits rotate around $\gamma^{\text {dou }}$. No homoclinics admit collisions, see Figure 3 .

(iii) Abbreviate by $\gamma_{M}^{\text {ext }}$ the exterior collision orbit near the Moon for a fixed $c \in\left(c_{h}, 0\right)$ and $\mu \neq 1 / 2$. It admits homoclinics which collide with the Earth. No homoclinics can collide with the Moon. Furthermore, any non-collision homoclinic rotates around the Earth precisely once, see Figure 4.

Remark. The satellite can rotate in both direction along all non-collision homoclinic orbits. This follows from the fact that the system is invariant under the anti-symplectic involution $(q, p) \mapsto$ $(q,-p)$.

Acknowledgements: I wish to express my deepest gratitude to Urs Frauenfelder for his encouragement. I also thank Holger Dullin for helpful comments, the unknown referee for valuable comments and the Institute for Mathematics of the University of Augsburg for providing a supportive research environment. This work was supported by Deutsche Forschungsgemeinschaft grants CI 45/8-1 and FR 2637/2-1.

\section{THE MOLECULE THEORY}

Let $(M, \omega)$ be a four-dimensional symplectic manifold and $H \in C^{\infty}(M, \mathbb{R})$ be a Hamiltonian. We assume that the Hamiltonian system $(M, \omega, H)$ is integrable and abbreviate by $F: M \rightarrow \mathbb{R}$ a first integral. By the Arnold-Liouville theorem, see for example [1], each compact connected component of regular common level sets of $H$ and $F$ is a two-dimensional torus, called a Liouville torus. Consequently, the manifold $M$ is foliated by regular leaves which are Liouville tori and singular leaves along which $d H$ and $d F$ are linearly dependent. This foliation is referred to as the Liouville foliation.

Let $\Sigma$ be a compact regular level set of $H$ which is a three-dimensional submanifold of $M$. By abuse of notation, we use the same symbol $F$ for the restriction $\left.F\right|_{\Sigma}$. We assume that $F$ is Morse-Bott, i.e., each connected component of the set of critical points of $F$, denoted by $\operatorname{crit} F$, is a submanifold of $\Sigma$ and the restriction of $F$ to a small transversal section to $\operatorname{crit} F$ is Morse. In order to describe the Liouville foliation on $\Sigma$, we recall the Fomenko-Zieschang invariant, see for example [2, 4]: Let $L$ be a singular leaf of the Liouville foliation and denote by $U(L)$ its small neighborhood. The neighborhood $U(L)$ is said to be Liouville equivalent to another neighborhood $U^{\prime}(L)$ of $L$ if there exists a homeomorphism $\varphi: U(L) \rightarrow U^{\prime}(L)$ which maps the leaves to the leaves. A Liouville equivalence class of small neighborhoods of $L$ is called an atom (associated to $L$ ). The number of singular leaves in an atom is called its complexity. In this section, we only consider simple atoms, i.e., atoms with complexity one. In view of the classification of atoms, a simple atom has the type either $A, B$, or $A^{*}$, which are described in the following:

(i) the atom $A$ is the solid torus $S^{1} \times D^{2}$ whose core $S^{1} \times\{0\}$ is the singular fiber. This atom corresponds to either the maximum or minimum of the Morse-Bott integral $F$ and describes birth or death of a Liouville torus;

(ii) the atom $B$ is the direct product of a neighborhood $\mathcal{N}$ of the figure eight and the circle, where the product of the figure eight and the circle represents the singular fiber. This atom is associated to saddle points of $F$ and describes the decomposition of a Liouville torus into two tori or the reverse;

(iii) the atom $A^{*}$ is obtained by glueing the endpoints of the product $\mathcal{N} \times[0,1]$ via the involution $I$ given in Figure 5 in such a way that $(x, 0)$ and $(I(x), 1)$ are identified. This atom is also 

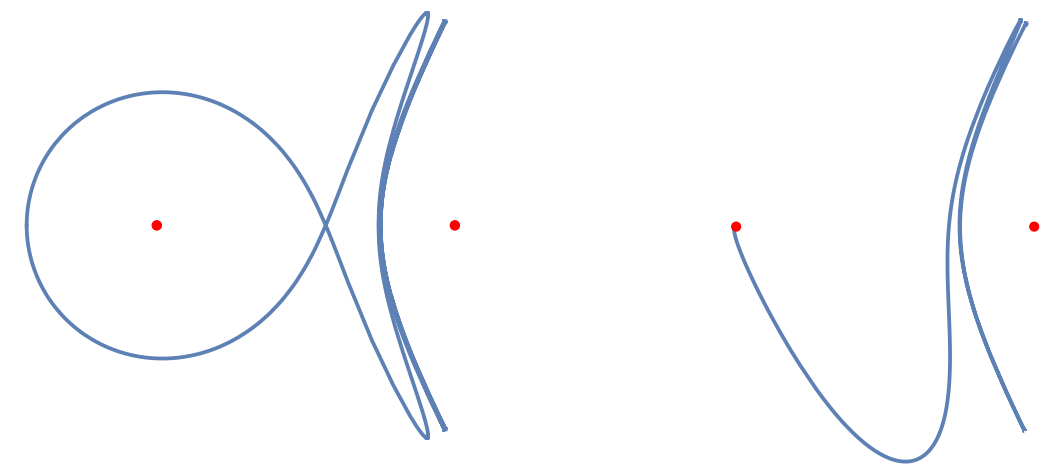

FIgURE 2. Noncollision and collision homoclinic orbits to the hyperbolic orbit

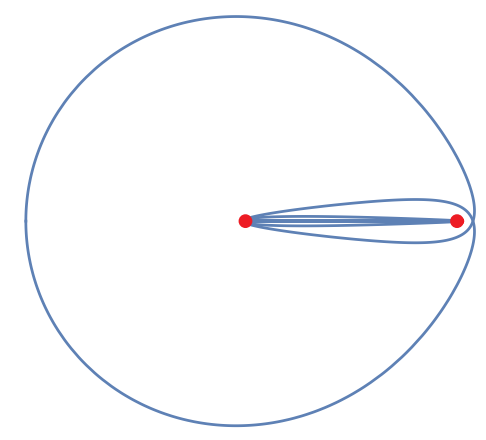

FiguRE 3. A homoclinic orbit to the double-collision orbit
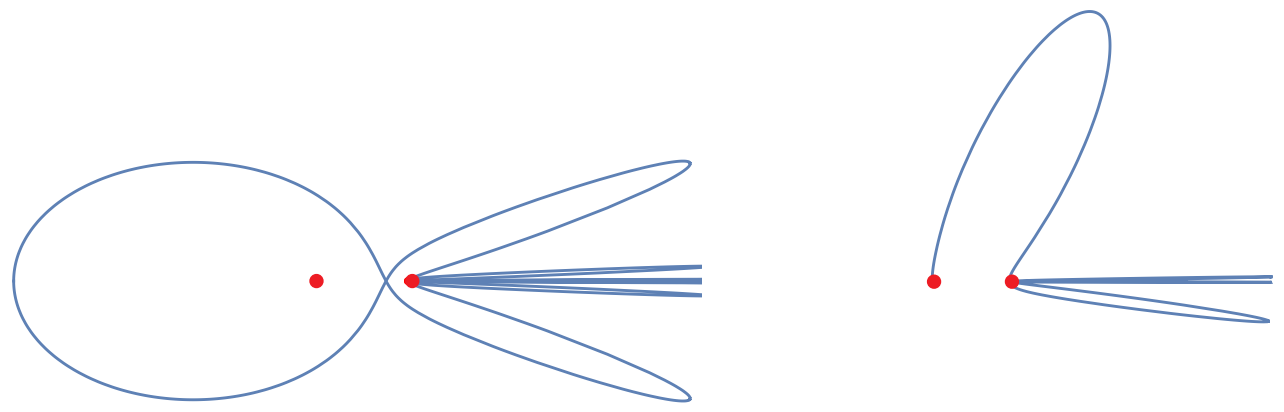

FiguRE 4. Noncollision and collision homoclinic orbits to the exterior collision orbit in the Moon component 


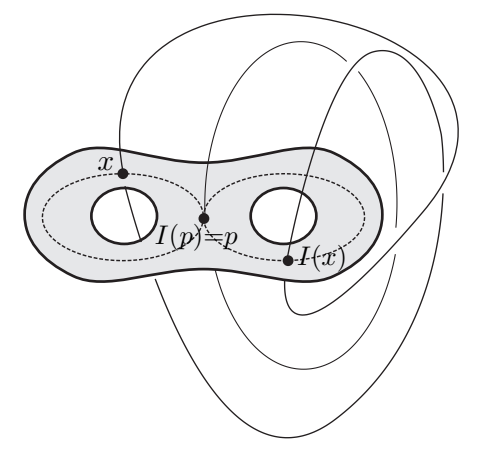

FIgURE 5. The neighrborhood $\mathcal{N}$ of the figure eight equipped with the involution $I$

associated to saddle points of $F$ and describes the transition from a Liouville torus into another one, see Figure 6 .

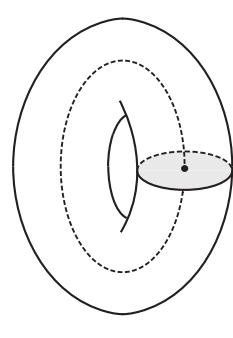

$A$
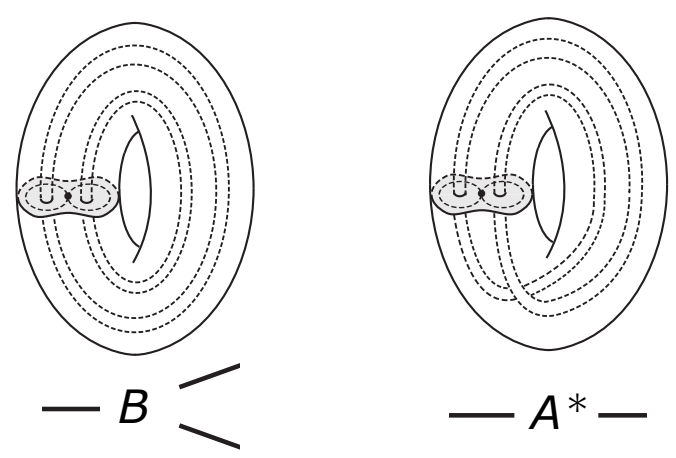

Figure 6. (left) the atom $A$, (middle) the atom $B$, (right) the atom $A^{*}$

In order to see topology of the three-dimensional manifold $\Sigma$ one needs to know how neighborhoods of singular fibers are glued along their boundaries. A combination of atoms of $\Sigma$ is called the molecule of $\Sigma$. Note that a molecule might describe several topologies. For example, if $\Sigma$ has the molecule $A-A$, then it can be obtained by glueing two solid tori along boundaries and hence it is homeomorphic to either $S^{3}, \mathbb{R} P^{3}, S^{1} \times S^{2}$, or lens spaces. To know the precise topology of $\Sigma$, one needs additional data, see [2, 4] and references cited therein.

\section{The Euler PROBlem OF TWO FIXED CENTERS}

In this section we recall some facts on the Euler problem. We introduce the doubly-covered elliptic coordinates $\left(\lambda, \nu, p_{\lambda}, p_{\nu}\right)$ which are defined by

$$
\cosh \lambda=|q-E|+|q-M| \in[1, \infty), \quad \cos \nu=|q-E|-|q-M| \in[-1,1],
$$

where $(\lambda, \nu) \in \mathbb{R} \times S^{1}[-\pi, \pi]$. Note that coordinate lines $\lambda=$ constant resp. $\nu=$ constant represent ellipses resp. hyperbola in the $q$-plane. The momenta $p_{\lambda}$ and $p_{\nu}$ are determined by the canonical relation $p_{1} d q_{1}+p_{2} d q_{2}=p_{\lambda} d \lambda+p_{\nu} d \nu$ and then the Hamiltonian becomes

$$
H=\frac{H_{\lambda}+H_{\nu}}{\cosh ^{2} \lambda-\cos ^{2} \nu},
$$


where $H_{\lambda}=2 p_{\lambda}^{2}-2 \cosh \lambda$ and $H_{\nu}=2 p_{\nu}^{2}+2(1-2 \mu) \cos \nu$. A Morse-Bott integral is given by

$$
G=-\frac{H_{\lambda} \cos ^{2} \nu+H_{\nu} \cosh ^{2} \lambda}{\cosh ^{2} \lambda-\cos ^{2} \nu} .
$$

Given $(G, H)=(g, h)$ the momenta can be expressed by

$$
p_{\lambda}^{2}=\frac{c \cosh ^{2} \lambda+2 \cosh \lambda+g}{2}, \quad p_{\nu}^{2}=\frac{-c \cos ^{2} \nu-2(1-2 \mu) \cos \nu-g}{2} .
$$

In particular, the system becomes separable in the elliptic coordinates.

Given $H=c$, we define the new Hamiltonian

$$
Q=(H-c)\left(\cosh ^{2} \lambda-\cos ^{2} \nu\right)=2 p_{\lambda}^{2}-2 \cosh \lambda-c \cosh ^{2} \lambda+2 p_{\nu}^{2}+2(1-2 \mu) \cos \nu+c \cos ^{2} \nu .
$$

Note that orbits of $H$ with energy $c$ and the time parameter $t$ correspond to orbits of $Q$ with energy 0 and the time parameter $\tau$, which is defined by

$$
\tau=\int \frac{d t}{\cosh ^{2} \lambda-\cos ^{2} \nu}
$$

Moreover, each bounded component of the energy hypersurface $H^{-1}(c)$ compactifies to each compact component, which is diffeomorphic to $S^{3}$, of the regularized hypersurface $Q^{-1}(0)$. In particular, the satellite is allowed to pass through the primaries. In the following we assume that the system is regularized.

We now define the function

$$
f_{\mu}(x)=c x^{2}+2(1-2 \mu) x+g
$$

so that $p_{\lambda}= \pm \sqrt{f_{0}(\cosh \lambda) / 2}$ and $p_{\nu}= \pm \sqrt{-f_{\mu}(\cos \nu) / 2}$. The classically allowed values $(g, c)$ are those at which the momenta are real. Note that for the momenta to be real we need $f_{0}>0$ for $x \in[1, \infty)$ and $f_{\mu}<0$ for $\mu \neq 0$ and $x \in[-1,1]$. Abbreviate by $x_{1}^{\mu}, x_{2}^{\mu}$ two roots of $f_{\mu}$. In the following we describe the procedure to find all such values in the lower half $(g, c)$-plane, which is given for example in [6, 8].

Case 1. the function $f_{\mu}$ has no real roots.

In this case we have $(1-2 \mu)^{2}<g c$ and $f_{\mu}<0$.

Case 2. the function $f_{\mu}$ admits a common root $x^{\mu}$. This implies that $(1-2 \mu)^{2}=g c$ and $x=-(1-2 \mu) / c$.

Case 3. the function $f_{\mu}$ has two real roots $x_{1}^{\mu}<x_{2}^{\mu}$. In the following we omit the obvious condition $(1-2 \mu)^{2}>g c$.

(i) $x_{1}^{\mu}<x_{2}^{\mu}<-1<1$ : since $x_{2}^{\mu}=\left(-(1-2 \mu)-\sqrt{(1-2 \mu)^{2}-g c}\right) / c$ is positive, this is impossible;

(ii) $x_{1}^{\mu}<-1<x_{2}^{\mu}<1 \Leftrightarrow 2(1-2 \mu)<c+g<-2(1-2 \mu)$ which is impossible;

(iii) $x_{1}^{\mu}<-1<1<x_{2}^{\mu} \Leftrightarrow 0<c+g-2(1-2 \mu)$;

(iv) $-1<x_{1}^{\mu}<x_{2}^{\mu}<1 \Leftrightarrow c+g+2(1-2 \mu)<0$ with $c<-(1-2 \mu)$;

(v) $-1<x_{1}^{\mu}<1<x_{2}^{\mu} \Leftrightarrow-2(1-2 \mu)<c+g<2(1-2 \mu)$;

(vi) $-1<1<x_{1}^{\mu}<x_{2}^{\mu} \Leftrightarrow c+g+2(1-2 \mu)<0$ with $c>-(1-2 \mu)$;

(vii) $x_{1}^{\mu}=-1<x_{2}^{\mu}<1 \Leftrightarrow 4(1-2 \mu)<0$ which is impossible;

(viii) $x_{1}^{\mu}=-1<x_{2}^{\mu}=1 \Leftrightarrow c+g= \pm 2(1-2 \mu)$ which is impossible;

(ix) $x_{1}^{\mu}=-1<1<x_{2}^{\mu} \Leftrightarrow c+g-2(1-2 \mu)=0$ if $\mu \neq 1 / 2$;

(x) $x_{1}^{\mu}<-1<x_{2}^{\mu}=1 \Leftrightarrow 0<-4(1-2 \mu)$ which is impossible;

(xi) $-1<x_{1}^{\mu}<x_{2}^{\mu}=1 \Leftrightarrow c+g+2(1-2 \mu)=0$ with $c<-(1-2 \mu)$;

(xii) $-1<1=x_{1}^{\mu}<x_{2}^{\mu} \Leftrightarrow c+g+2(1-2 \mu)=0$ with $c>-(1-2 \mu)$.

In view of the fact that for the momenta to be real we need $f_{0}>0$ with $x \in[1, \infty)$ and $f_{\mu}<0$, $\mu \neq 0$, with $x \in[-1,1]$, we combine the above results and give the bifurcation diagram in Table 1 and Figure 7 


\begin{tabular}{|c|c|c|}
\hline Region & Ranges of the Roots & Ranges of the Variables \\
\hline$P$ & $\begin{aligned} \cosh \lambda & :-1<1<x_{1}^{0}<x_{2}^{0} \\
\cos \nu & :\left\{\begin{array}{cc}-1<1<x_{1}^{\mu}<x_{2}^{\mu}, & (1-2 \mu)^{2} \geq g c \\
\text { complex roots, } & (1-2 \mu)^{2}<g c\end{array}\right.\end{aligned}$ & $\begin{aligned} \cosh \lambda & \in\left[x_{1}^{0}, x_{2}^{0}\right] \\
\cos \nu & \in[-1,1]\end{aligned}$ \\
\hline$L$ & $\begin{aligned} \cosh \lambda & :-1<x_{1}^{0}<1<x_{2}^{0} \\
\cos \nu & :\left\{\begin{array}{cc}-1<1<x_{1}^{\mu}<x_{2}^{\mu}, & (1-2 \mu)^{2} \geq g c \\
\text { complex roots, } & (1-2 \mu)^{2}<g c\end{array}\right.\end{aligned}$ & $\begin{array}{l}\cosh \lambda \in\left[1, x_{2}^{0}\right] \\
\cos \nu \in[-1,1]\end{array}$ \\
\hline$S$ & $\begin{array}{l}\cosh \lambda:-1<x_{1}^{0}<1<x_{2}^{0} \\
\cos \nu:-1<x_{1}^{\mu}<x_{2}^{\mu}<1\end{array}$ & $\begin{array}{c}\cosh \lambda \in\left[1, x_{2}^{0}\right] \\
\cos \nu \in\left(\left[-1, x_{1}^{\mu}\right] \cup\left[x_{2}^{\mu}, 1\right]\right)\end{array}$ \\
\hline$S^{\prime}$ & $\begin{array}{l}\cosh \lambda:-1<x_{1}^{0}<1<x_{2}^{0} \\
\cos \nu:-1<x_{1}^{\mu}<1<x_{2}^{\mu}\end{array}$ & $\begin{array}{l}\cosh \lambda \in\left[1, x_{2}^{0}\right] \\
\cos \nu \in\left[-1, x_{1}^{\mu}\right]\end{array}$ \\
\hline
\end{tabular}

TABLE 1. The ranges of the roots and the variables in the four regular regions

As mentioned in the introduction, if $\mu \neq 1 / 2$, in the lower-half $(G, H)=(g, c)$-plane there exist four regular regions consisting of regular values of the energy momentum mapping $(\lambda, \nu) \mapsto$ $(G(\lambda, \nu), H(\lambda, \nu))$. By the Arnold-Liouville theorem, each point in the regular regions represents a Liouville torus. Following the notations from [3, 5, the regular regions are labeled by $S^{\prime}, S$ (satellite), $L$ (lemniscate), and $P$ (planetary). They are bounded by the following five critical curves:

$$
\begin{array}{ll}
\ell_{1,2}: c=-g \pm 2(1-2 \mu), & \ell_{3}: c=-g-2, \\
\ell_{4}: g c=(1-2 \mu)^{2}, c_{J}<c<c_{h}, & \ell_{5}: g c=1, c_{e}<c .
\end{array}
$$

Here, $c_{e}=-1$ or $c_{h}=-(1-2 \mu)$ are the energy levels at which the line $\ell_{3}$ and curve $\ell_{5}$ or the line $\ell_{2}$ and curve $\ell_{4}$ intersect, respectively(the letters $e$ and $h$ stand for elliptic and hyperbolic). Note that at these points the Liouville foliation changes. If the two primaries have the equal masses, $\mu=1 / 2$, then the two curves $\ell_{1}, \ell_{2}$ become equal and hence the $S^{\prime}$-region does not appear. In the $S$-region each point represents a motion of the satellite which is confined to a neighborhood of either the Earth or Moon, while in the $S^{\prime}$-region the motion takes place only near the Earth, see for example [6. 8].

We now examine the critical orbits:

(i) On $l_{1}: c=-g+2(1-2 \mu)$. The satellite moves along the ray $\cos \nu=-1$, but the motion is bounded by the ellipse $\cosh \lambda=x_{2}^{0}$. We call this orbit the exterior collision orbit in the Earth component;

(ii) $O n l_{2}: c=-g-2(1-2 \mu)$. Each point on the line $l_{2}$ represents an orbit in a neighborhood of either the Earth or Moon. Assume the satellite moves near the Earth so that $(\cosh \lambda, \cos \nu) \in$ $\left[1, x_{2}^{0}\right] \times\left[-1, x_{1}^{\mu}\right]$. In particular, the motion is regular. Consider orbits near the Moon. Then the satellite moves along the ray $\cos \nu=1$, but bounded by the ellipse $\cosh \lambda=x_{2}^{0}$. This orbit is called the exterior collision orbit in the Moon component;

(iii) $\mathrm{On} \mathrm{l}_{3}: c=-g-2$. On the line $l_{3}$ we have $\cosh \lambda=1$, i.e, the satellite moves along the line segment joining the two masses. If $c<c_{J}$, the variable $\cos \nu$ lies in $\left[-1, x_{1}^{\mu}\right] \cup$ $\left[x_{2}^{\mu}, 1\right]$, where the first or the second interval corresponds to the motion in the Earth or 


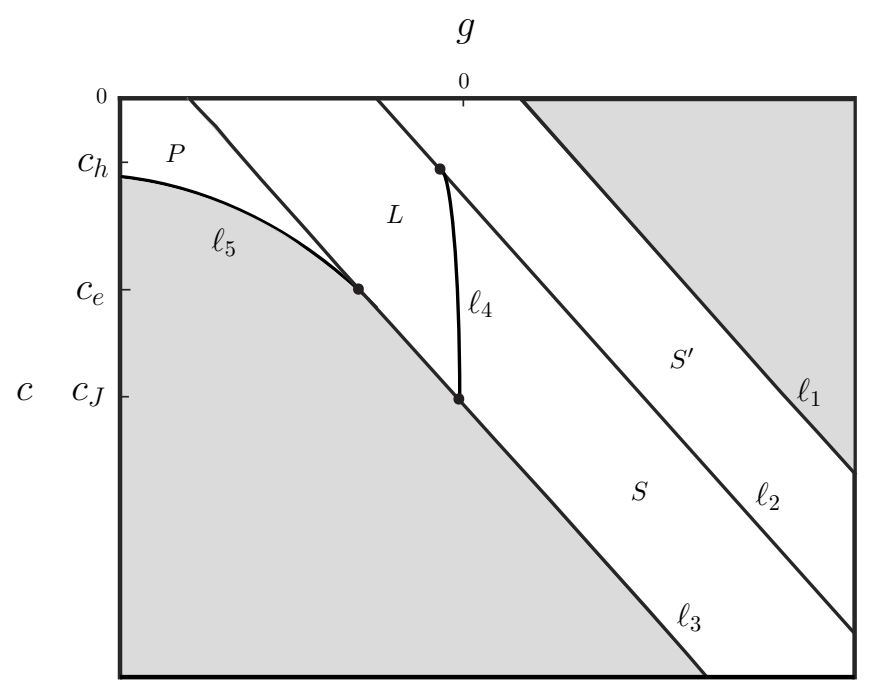

Figure 7 . The case $\mu \neq 1 / 2$ : The lower half $(g, c)$-plane is divided into four regions by the five critical curves. The shaded regions are classically not allowed. For negative energies, each regular level of the energy-momentum mapping represents one of four types of motions, which are labeled by $S^{\prime}, S, L$ and $P$.

Moon component, respectively. For $\cos \nu \in\left[-1, x_{1}^{\mu}\right]$, the satellite moves on the line segment $\left\{\left(q_{1}, 0\right):-1 / 2 \leq q_{1} \leq b_{c}^{E}\right\}$, where $\left(b_{c}^{E}, 0\right) \in \partial \mathcal{K}_{c}^{E}$ with $b_{c}^{E} \in(-1 / 2, l)$. Similarly, in the Moon component we have $\left\{\left(q_{1}, 0\right): b_{c}^{M} \leq q_{1} \leq 1 / 2\right\}$, where $\left(b_{c}^{M}, 0\right) \in \partial \mathcal{K}_{c}^{M}$ with $b_{c}^{M} \in(l, 1 / 2)$. We refer to such an orbit as the interior collision orbit in the Earth or Moon component. As $c$ passes over $c_{J}$, the two components of the Hill's region become connected. The two interior collision orbits also become connected so that the satellite can move between the Earth and Moon. Indeed, there is no restriction on $\cos \nu$, i.e., $\cos \nu \in[-1,1]$ for $c>c_{J}$. This orbit is referred to as the double-collision orbit;

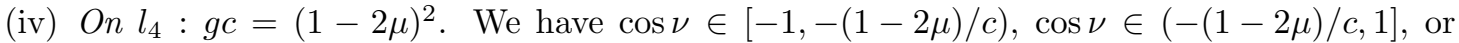
$\cos \nu=-(1-2 \mu) / c$. For the last case, the satellite moves along the hyperbola $\cos \nu=$ $-(1-2 \mu) / c$ within the ellipse $\cosh \lambda=x_{-}^{0}$. We call this orbit the hyperbolic orbit. Each member of the family $\gamma_{\text {hyp }}^{c}$ of the hyperbolic orbits, $c \in\left(c_{J}, c_{h}\right)$, is the Lyapunov orbit. To see this, we need to show that the family $\gamma_{\text {hyp }}^{c}$ converges uniformly to $L=(l, 0,0,0)$ as $c$ tends to $c_{J}$ from above, i.e, it emanates from the critical point. Recall that along the hyperbolic orbits the equation $c \cos \nu^{2}+2(1-2 \mu) \cos \nu+g=0$ has double roots $\cos \nu=-(1-2 \mu) / c$ which implies that the hyperbola is given by

$$
\left|q+\left(\frac{1}{2}, 0\right)\right|-\left|q-\left(\frac{1}{2}, 0\right)\right|=-\frac{1-2 \mu}{c} .
$$

This hyperbola, for $\mu \neq 1 / 2$, is closer to the Moon than to the Earth since $-(1-2 \mu) / c>0$. It remains to show that the hyperbola $\cos \nu=-(1-2 \mu) / c$ consists of the single point $(l, 0)$ at $c=c_{J}$, or equivalently

$$
\left\{\left(q_{1}, q_{2}\right):\left|q+\left(\frac{1}{2}, 0\right)\right|-\left|q-\left(\frac{1}{2}, 0\right)\right|=-\frac{1-2 \mu}{c_{J}}\right\} \cap \mathcal{K}_{c_{J}}=\{(l, 0)\} .
$$

Since $\cosh \lambda \rightarrow 1$ as $c \rightarrow c_{J}$, it suffices to show that the vertex of this hyperbola is given by $(l, 0)$. We compute that 


$$
\frac{1-2 \mu}{-c_{J}}=\frac{1-2 \mu}{1+2 \sqrt{\mu(1-\mu)}}=\frac{1-2 \sqrt{\mu(1-\mu)}}{1-2 \mu} .
$$

Then the vertex of this hyperbola is given by

$$
\left(q_{1}, q_{2}\right)=\left(\frac{1-2 \sqrt{\mu(1-\mu)}}{2(1-2 \mu)}, 0\right)=(l, 0) .
$$

This completes the proof of the claim. On the other hand, as $c \rightarrow c_{h}$ from below the hyperbolic orbits degenerates to the exterior collision orbit in the Moon component;

(v) On $l_{5}: g c=1$. We have $\cosh \lambda=-1 / c$, but no restriction on $\cos \nu$. This implies that the satellite moves along the ellipse $\cosh \lambda=-1 / c$. This orbit is referred to as the elliptic orbit. As $c \rightarrow c_{e}$ from above, the ellipse $\cosh \lambda=-1 / c$ degenerates to the line segment $\cosh \lambda=1$ joining the two masses, namely the double-collision orbit.

\section{Molecules of the Euler Problem}

The discussion in the previous section together with the results of the molecule theory described in Section 2 give rise to the molecules of the Euler problem, which are already given by WaalkensDullin-Richter in [8, Section 2], see Figure 8, We refer to [7] for the molecule structure of the Euler problem on the two-sphere.
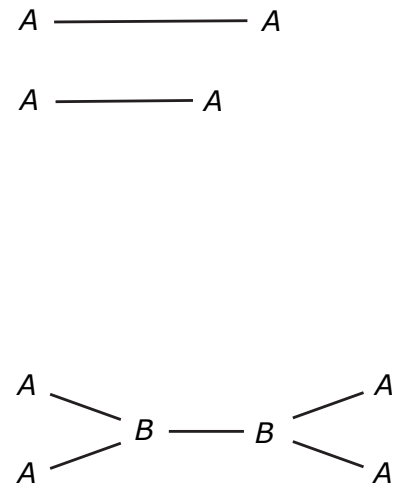
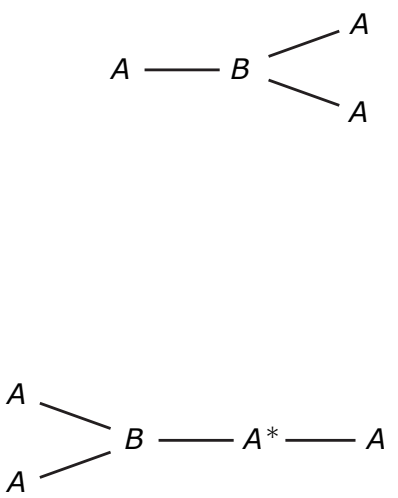

Figure 8. Molecules of the Euler problem. (left above) For $c<c_{J}$ : The above one is associated to the Earth component and the below one is associated to the Moon component. The leftmost $A$-atoms represent the interior collision orbits and the rightmost ones represent the exterior collision orbits. (right above) For $c_{J}<c_{e}$ : The leftmost $A$ represents the double-collision orbit and the middle $B$ is the hyperbolic orbit. The right two atoms are exterior collision orbits in both components. (left below) For $c_{e}<c<c_{h}$ : The leftmost atoms are the elliptic orbits. The first, from the left, atom $B$ is the double-collision orbit and the second one is the hyperbolic orbit. The rightmost atoms are the exterior collision orbits. (right below) For $c_{h}<c<0$ : As in the previous case, the first two atoms are the elliptic orbits and the atom $B$ is the double collision orbit. In this energy range, the hyperbolic orbit does not appear and the exterior collision orbit in the Moon component becomes unstable: the atom $A^{*}$. The last atom $A$ represents the exterior collision orbit in the Earth component. 


\section{The EXISTENCE OF HOMOCLINIC ORBITS}

Recall that in the Euler problem the hyperbolic orbit $\gamma^{\text {hyp }}$ for $c \in\left(c_{J}, c_{h}\right)$, the double-collision orbit $\gamma^{\text {dou }}$ for $c \in\left(c_{e}, 0\right)$ and the exterior collision orbit in the Moon component $\gamma_{M}^{\text {ext }}$ for $c \in\left(c_{h}, 0\right)$, if $\mu \neq 1 / 2$, are unstable periodic orbits. The existence of homoclinic orbits to each of these orbits follows immediately from the properties of the corresponding atoms as follows:

Case 1. The hyperbolic orbit $\gamma^{\text {hyp }}$

Recall that the corresponding atom is the type $B$ whose singular fiber, denoted by $L$, is homeomorphic to the union of two two-dimensional tori attached along a single periodic orbit which is in this case the hyperbolic orbit $\gamma^{\text {hyp }}$. A manifold $L \backslash \gamma^{\text {hyp }}$ consists of two connected components, where each of them is diffeomorphic to the two-torus minus a periodic orbit: one is around the Earth and the other is around the Moon. Here we consider only the component near the Earth, which is abbreviated by $L_{E} \cong \mathbb{T}^{2} \backslash \gamma^{\text {hyp }}$. For the component near the Moon, the same argument also holds. Since $\gamma^{\text {hyp }}$ is periodic, it has some rational slope on the torus. We then observe that on $L_{E}$ there exist no periodic orbits. Otherwise, all periodic orbits have the same rational slope with $\gamma^{\text {hyp }}$, but this is not the case in view of the symmetry associated with the integral $G$. Therefore, every orbit on $L_{E}$ is of irrational slope and hence is dense. This implies that every orbit converges asymptotically to $\gamma^{\text {hyp }}$ in both directions, namely all orbits on $L_{E}$ are homoclinic orbits.

Case 2. The double-collision orbit $\gamma^{\text {dou }}$

In this case we also have the atom $B$. The argument given in the previous case carries over to this case and hence there exist homoclinic orbits to $\gamma^{\text {dou }}$.

Case 3. The exterior collision orbit in the Moon component $\gamma_{M}^{\text {ext }}$

We have the atom $A^{*}$. The singular fiber $L$ is given by the "skew product" of the figure eight and the circle via the involution given in Figure 5. Note that $L \backslash \gamma_{M}^{\text {ext }}$ consists of a single connected component: $L \backslash \gamma_{M}^{\text {ext }} \cong \mathbb{T}^{2} \backslash \gamma^{\text {hyp }}$. The remaining argument is the same as before.

Remark 5.1. The above discussions on the existence of homoclinic orbits only make use of the existence of the atoms $B$ and $A^{*}$. Therefore, for integrable Hamiltonian systems with two degrees of freedom, any unstable periodic orbit associated to the atom $B$ or $A^{*}$ admits homoclinic orbits.

\section{Proof of the theorem}

Since homoclinic orbits to a unstable periodic orbit $\gamma$ lie on the intersection of the unstable and stable manifolds of $\gamma$, if they exist, then they take the same integral value as $\gamma$.

Case 1. The hyperbolic orbit $\gamma^{\text {hyp }}$

Let us fix the energy level $c \in\left(c_{J}, c_{h}\right)$ at which $\gamma^{\text {hyp }}$ exists. Recall from Section 3 that the corresponding value $(g, c)$ lies on $\ell_{4}$ so that $\gamma^{\text {hyp }}$ takes $g_{c}^{\text {hyp }}:=(1-2 \mu)^{2} / c<0$.

We first consider homoclinic orbits along which collisions do not occur and observe the behavior of the satellite along these orbits. In view of the argument in Section 3 , such orbits lie in a neighborhood of the Earth or Moon in the case $\cos \nu \in[-1,-(1-2 \mu) / c)$ or $\cos \nu \in(-(1-2 \mu) / c, 1]$, respectively, where $\cos \nu=-(1-2 \mu) / c$ represents $\gamma^{\text {hyp }}$. In $(\lambda, \nu)$-coordinates, the range for $\nu$ is given by $\left[-\pi,-\nu_{1}\right) \cup\left(\nu_{1}, \pi\right]$, where $\cos \nu_{1}=-(1-2 \mu) / c$. Without loss of generality we only consider the interval $\left[-\pi,-\nu_{1}\right)$. For $\left(\nu_{1}, \pi\right]$, the satellite shows the same behavior.

By the Hamiltonian equations and (1) we have

$$
\begin{aligned}
\dot{\nu} & =\frac{\partial H}{\partial p_{\nu}} \\
& =\frac{4 p_{\nu}}{\cosh ^{2} \lambda-\cos ^{2} \nu} \\
& = \pm \frac{4}{\cosh ^{2} \lambda-\cos ^{2} \nu} \sqrt{\frac{c^{2} \cos ^{2} \nu+2(1-2 \mu) c \cos \nu+(1-2 \mu)^{2}}{-2 c}}
\end{aligned}
$$




$$
= \pm \frac{2 \sqrt{2} \sqrt{-c}}{\cosh ^{2} \lambda-\cos ^{2} \nu}\left(\cos \nu+\frac{1-2 \mu}{c}\right) .
$$

Since we are considering non-collision orbits, the denominator $\cosh ^{2} \lambda-\cos ^{2} \nu$ never vanishes. Therefore, the sign of $\dot{\nu}$ remains unchanged unless $\nu= \pm \nu_{1}$. Thus, we conclude that $\dot{\nu}$ is nonvanishing. We also have

$$
\dot{\lambda}= \pm \frac{4}{\cosh ^{2} \lambda-\cos ^{2} \nu} \sqrt{\frac{c^{2} \cosh ^{2} \lambda+2 c \cosh \lambda+(1-2 \mu)^{2}}{2 c}} .
$$

Note that $c^{2} \cosh ^{2} \lambda+2 c \cosh \lambda+(1-2 \mu)^{2}$ is negative for $\cosh \lambda \in\left[-1, c_{J} / c\right]$ which shows that $\dot{\lambda}$ is also nonvanishing. Therefore, $\cosh \lambda$ oscillates in the interval $\left[-1, c_{J} / c\right]$.

Suppose that $\nu=\nu_{0} \in\left(-\pi,-\nu_{1}\right)$ and $\dot{\nu}>0$ at $t=t_{0}$. Then by the previous argument, $\nu$ converges asymptotically to $\nu=-\nu_{1}$ as $t \rightarrow \infty$. On the other hand, in backward time, i.e., as $t$ decreases, $\nu$ also converges asymptotically to $\nu=-\nu_{1}$. Since $\nu$ is defined on the circle $S^{1}[-\pi, \pi]$ this picture holds true. We obtain a similar picture if we start with $\dot{\nu}<0$ at $t=t_{0}$.

Along each non-collision homoclinic orbit the variable $\nu$ attains $-\pi$ precisely once. In $\left(q_{1}, q_{2}\right)$ coordinates, this implies that the satellite crosses the subset $K$ of the negative $q_{1}$-axis, which is the line segment joining the boundary of the Hill's region $\mathcal{K}_{c}^{E}$ and the Earth, precisely once. To prove the assertion that every non-collision homoclinic orbit rotates around one of the two primaries precisely once, it remains to show that the trajectory cannot be tangent to $K$. Suppose that the tangency occurs at $t=t^{\prime}$ and hence $\dot{\nu}=0$. By the equation 22 , that $\dot{\nu}=0$ is equivalent to that $c=c_{h}=-1+2 \mu$, which contradicts to $c \in\left(c_{J}, c_{h}\right)$, see Figure 2 .

To show the existence of collision homoclinic orbits, we need to show that its projection to the configuration space contains the two primaries at which we have $\cosh \lambda=1, \cos \nu= \pm 1$. It then suffices to show that for a fixed $g=(1-2 \mu)^{2} / c, c \in\left(c_{J}, c_{h}\right)$ and $(\cosh \lambda, \cos \nu)=(1, \pm 1)$, the squared momenta (1) are positive. To see this, we first compute that

$$
\begin{aligned}
p_{\lambda}^{2} & =\frac{c \cosh ^{2} \lambda+2 \cosh \lambda+g}{2} \\
& =\frac{c+2+(1-2 \mu)^{2} / c}{2} \\
& =\frac{c^{2}+2 c+(1-2 \mu)^{2}}{2 c} .
\end{aligned}
$$

Since $c_{h}=-1+2 \mu \leq-1+2 \sqrt{\mu(1-\mu)}$ in view of $\mu \leq 1 / 2$, we obtain that $c^{2}+2 c+(1-2 \mu)^{2}$ is negative for $c \in\left(c_{J}, c_{h}\right)$ which implies that $p_{\lambda}^{2}$ is positive. We also compute that

$$
\begin{aligned}
p_{\nu}^{2} & =\frac{-c \cos ^{2} \nu-2(1-2 \mu) \cos \nu-g}{2} \\
& =\frac{-c \pm 2(1-2 \mu)-(1-2 \mu)^{2} / c}{2} \\
& =\frac{(c \pm(1-2 \mu))^{2}}{-2 c}>0 .
\end{aligned}
$$

This proves the assertion on the existence of collision homoclinic orbits, see Figure 2 .

Case 2. The double-collision orbit $\gamma^{\text {dou }}$

We fix $c \in\left(c_{e}, 0\right)$. Recall that $\gamma^{\text {dou }}$ takes the value $g_{c}^{\text {dou }}:=-c-2 \in(-2,-1)$. Note that

$$
(\lambda, \nu) \mapsto\left(q_{1}, q_{2}\right)=\left(\frac{1}{2} \cosh \lambda \cos \nu, \frac{1}{2} \sinh \lambda \sin \nu\right)
$$

is a 2-to- 1 covering with two branch points $E$ and $M$. The two sheets are related by the involution $(\lambda, \nu) \mapsto(-\lambda,-\nu)$ which extends to the phase space by

$$
\left(\lambda, \nu, p_{\lambda}, p_{\nu}\right) \mapsto\left(-\lambda,-\nu,-p_{\lambda},-p_{\nu}\right) .
$$


Any homoclinic orbit to $\gamma^{\text {dou }}$ has $\cosh \lambda \in\left(1, \cosh \lambda_{1}\right]$, where $\lambda_{1}>0$ satisfies $\cosh \lambda_{1}=(-2-c) / c$. Hence we have $\lambda \in\left[-\lambda_{1}, 0\right) \cup\left(0, \lambda_{1}\right]$. As a result, the two connected components of the singular fiber minus $\gamma^{\text {dou }}$ are related to each other via the involution (3). Therefore, to observe the behavior of the satellite along homoclinic orbits, without loss of generality, we may consider only $\lambda \in\left(0, \lambda_{1}\right]$.

Following an argument similar with the one given in the previous case one can easily see that there exist no collision homoclinic orbits to $\gamma^{\text {dou }}$. In view of the Hamiltonian equations and (1) along homoclinics we have

$$
\dot{\lambda}= \pm \frac{4}{\cosh ^{2} \lambda-\cos ^{2} \nu} \sqrt{\frac{c \cosh ^{2} \lambda+2 \cosh \lambda-c-2}{2}}
$$

which shows that $\dot{\lambda}=0$ if and only if $\lambda=\lambda_{1}$. Suppose that $\lambda \in\left(1, \lambda_{1}\right]$ and $\dot{\lambda}>0$ at $t=t_{0}$. In forward time, $\dot{\lambda}$ remains to be positive until $\lambda=\lambda_{1}$ so that $\lambda$ increases. At $\lambda=\lambda_{1}$ the velocity $\dot{\lambda}$ vanishes and then becomes negative. As time further increases, $\lambda$ decreases and converges asymptotically to $\lambda=1$. In backward time $\lambda$ also converges asymptotically to $\lambda=1$. We obtain a similar picture for the other case.

Note that there are no constraints on the variable $\nu$, i.e., $\nu \in S^{1}[-\pi, \pi]$. As in the previous case, one can easily show that $\dot{\nu}$ is nonvanishing along homoclinics. As a result, $\dot{\nu}$ is either always positive or always negative which shows that along homoclinic orbits $\cos \nu$ oscillates in the interval $[-1,1]$. This together with the previous discussion show that homoclinic orbits rotate around the double-collision orbit, see Figure 3 .

Case 3. The exterior collision orbit in the Moon component $\gamma_{M}^{e x t}$

We fix $\mu<1 / 2$ and $c \in\left(c_{h}, 0\right)$. Abbreviate $g_{c}^{\text {ext }}=-c-2(1-2 \mu)$. In this case we have $\cos \nu \in[-1,1)$, where $\cos \nu=1$ represents $\gamma_{M}^{\text {ext }}$. The range for $\nu$ is then given by $[-\pi, 0) \cup(0, \pi]$. Without loss of generality, we only consider $[-\pi, 0)$.

By the same reasoning as in the first case with $\nu_{1}=0$, we obtain that $\dot{\lambda}$ and $\dot{\nu}$ are nonvanishing along noncollision homocilinics and hence $\lambda$ oscillates and $\nu$ converges asymptotically to 0 in backward and forward time. Moreover, each non-collision homoclinic crosses the line segment, which is a subset of the negative $q_{1}$-axis and which joins the Earth and the boundary $\partial \mathcal{K}_{c}$, precisely once. We also obtain that there exist homoclinics which collide with the Earth. There exist no collisions with the Moon, see Figure 4 .

\section{REFERENCES}

[1] V. I. Arnold, Mathematical methods of classical mechanics, Graduate texts in mathematics 60, New York, Springer, 2000.

[2] A. V. Bolsinov and A. T. Fomenko, Integrable Hamiltonian systems: Geometry, Topology, Classification, Chapman \& Hall/CRC, 2004.

[3] C. L. Charlier, Die Mechanik des Himmels, Veit \& Comp., Leipzig (1902).

[4] A. T. Fomenko and H. Zieschang, A topological invariant and a criterion for the equivalence of integrable Hamiltonian systems with two degrees of freedom, Izv. Akad. Nauk. SSSR. Ser. Matem. 54 no. 3 (1990), 546-572.

[5] W. Pauli, Über das Modell des Wasserstoffmolekülions, Annalen der Physik 68 (1922), 177-240.

[6] M. P. Strand and W. P. Reinhardt, Semiclassical quantization of the low lying electronic states of $H_{2}^{+}, J$. Chem. Phys. 70 (1979) 3812-3827.

[7] T. G. Vozmishcheva and A. A. Oshemkov, Topological analysis of the two-centre problem on the two-dimensional sphere, Sb. Math. 193 no. 8 (2002), 1103-1138.

[8] H. Waalkens, H. R. Dullin, and P. H. Richter, The problem of two fixed centers: bifurcations, actions, monodromy, Physica D. 196(3-4) (2004) 265-310.

Universität Augsburg, Universitätsstrasse 14, D-86159 Augsburg, Germany

E-mail address: seongchan.kim@math.uni-augsburg.de 